\title{
Propiedades físicas y mecánicas de la madera de Pinus canariensis crecido en el secano de la Región del Maule, Chile
}

\author{
Wood physical and mechanical properties of Pinus canariensis grown on the \\ dry lands in Maule's Region, Chile \\ Claudio Rodríguez ${ }^{\mathrm{a} *}$, Emilio Vergara ${ }^{\mathrm{b}}$ \\ *Autor de correspondencia: a Universidad Católica del Maule, Departamento de Ciencias Forestales, casilla 617, Talca, Chile, \\ crodrig@ucm.cl \\ b Universidad del Bío Bío, Concepción, Chile.
}

\begin{abstract}
SUMMARY
Physical and mechanical properties were evaluated in 46 year-old Pinus canariensis wood, growing in dry lands of Maule's Region in Chile. The wood was conditioned to $12 \%$ moisture content and mechanical testing was according to Chilean standards. The basic density was $0.503 \pm 0.03 \mathrm{~g} / \mathrm{cm}^{3}$. The results of mechanical properties were lower compared with those gotten in Spain and Australia. Concerning Pinus radiata wood, properties were within the range or over, except for perpendicular tension strength. According to chilean standard NCh1989, P. canariensis wood is classified in ES4 group, as native woods like coigüe, laurel, lenga and roble. Because of its structural properties, according to Sallenave classifications, this species is specially appropriated for building and other structural applications.
\end{abstract}

Key words: canary pine, dry lands, density, wood mechanical properties.

\section{RESUMEN}

En este estudio se evaluaron las propiedades físicas y mecánicas de la madera de Pinus canariensis de 46 años crecido en el secano de la Región del Maule, Chile. La madera se acondicionó a 12\% de humedad, y los ensayos físicos y mecánicos se efectuaron de acuerdo a las normas chilenas. La densidad básica alcanzó $0,503 \pm 0,03 \mathrm{~g} / \mathrm{cm}^{3}$. Las propiedades mecánicas resultaron inferiores a datos de la misma especie provenientes de España y Australia. Con relación al Pinus radiata crecido en el país, las determinaciones estuvieron dentro del rango o sobre su límite superior, con excepción de la resistencia a la tracción perpendicular. Según la norma chilena NCh1989, la madera de P. canariensis clasifica en el grupo de resistencia ES4, junto con nativas como coigüe, laurel, lenga y roble. Sus propiedades estructurales, conforme a la clasificación de Sallenave, la hacen especialmente atractiva para edificación y otras aplicaciones estructurales.

Palabras clave: pino canario, secano, densidad, propiedades mecánicas de la madera.

\section{INTRODUCCIÓN}

La búsqueda de especies forestales de rápido crecimiento, alternativas a las que hoy se cultivan en Chile, ha sido por décadas preocupación permanente de muchas organizaciones sectoriales competentes. La ejecución de diversos programas de introducción de especies es prueba de la importancia que el país le ha otorgado a la diversificación forestal como parte de la estrategia de desarrollo sustentable (INFOR 1986, Barros 1997).

Una especie que ha demostrado adaptación a las condiciones que ofrece el secano de la Región del Maule es el Pinus canariensis C. Smith. (pino canario, pino tea), presente en las localidades de Chanco y Llico, en donde fuera empleada en la contención de dunas litorales hace más de un siglo, así como en diversos programas de introducción de especies y ensayos recientes (Albert 1908,
INFOR 1986, CONAF 1990, Barros 1997, Rodríguez y Bobadilla 2006).

Los crecimientos medios anuales en diámetro a la altura del pecho (DAP) y altura registrados por Rodríguez y Bobadilla (2006) en un rodal de pino canario con manejo en el Centro Experimental de Tanumé (Corporación Nacional Forestal, VI Región) fueron $1,08 \mathrm{~cm}$ y $0,77 \mathrm{~m}$ a la edad de 12 años, respectivamente. Para el rodal empleado en este estudio, localizado en un sitio cercano a la ciudad de Talca, estos crecimientos promedian los 0,64 cm/año para el DAP y los $0,50 \mathrm{~m} /$ año para la altura a los 46 años. Estos valores son comparables a los informados por Quiroz y Rojas (2003) en rodales entre 12 y 34 años de pino ponderosa (Pinus ponderosa Dougl. ex Laws) en la zona sur de Chile, con valores en el rango 0,57-1,47 cm/año para el DAP; y de 0,15$0,57 \mathrm{~m} / a$ ño para la altura. 
La especie es endémica de Islas Canarias donde, en función del contenido de resina de su madera, se reconocen dos tipos: "pino blanco" y "pino tea". La primera debe su nombre al blanco amarillento de la albura, en tanto que el segundo es siempre duramen de color pardo rojizo y de aspecto acaramelado, que una vez cepillada es de una gran belleza. El estado intermedio entre estos dos tipos se conoce como "pino riga" (Guindeo y García 1988).

Su durabilidad natural es excelente y puede clasificarse entre las mejores calidades de madera de pino. En las Islas Canarias existen piezas todavía sanas después de 200 años de exposición a la intemperie. Se la ha usado en postes, pies derechos, madera aserrada para construcción, carpintería y ebanistería, tabiques, contenedores, elementos exteriores de viviendas, tornería, marquetería y tableros (Peraza y López 1967, Webb et al. 1984).

Un estudio desarrollado por Raymond et al. (2004) en Australia, donde $P$. canariensis se cultiva en zonas de secano, determinó que a las aplicaciones antes señaladas se agrega la aptitud para pulpaje kraft, pulpaje termomecánico y tableros de fibra de densidad media. Las propiedades de la pulpa kraft son similares a las obtenidas con Pinus radiata D. Don, pero de inferior rendimiento. En pulpaje termomecánico, el gasto de energía resultó más elevado que para $P$. radiata.

El hallazgo en 1997 de un bosquete de $P$. canariensis adulto en las cercanías de la ciudad de Talca, Región del Maule, permitió formular el presente estudio cuyo objetivo es evaluar las características físicas y mecánicas de esta madera y deducir sus posibles aplicaciones.

\section{MÉTODOS}

La muestra se obtuvo de cinco árboles del estrato dominante del único rodal de la especie que se conoce haya crecido bajo las particulares condiciones del secano interior de la Región del Maule: 6-7 meses secos, 700$900 \mathrm{~mm} / \mathrm{año}$ y, en general, condiciones desfavorables para el establecimiento de plantaciones de rápido crecimiento (Schlatter et al. 2001).

El rodal compuesto por aproximadamente 120 individuos de 46 años se encuentra en el predio La Esperanza (Talca, $35^{\circ} 28^{\prime} \mathrm{S}, 71^{\circ} 38^{\prime} \mathrm{O}$ ), a una altitud de $160 \mathrm{~m}$ s.n.m., exposición Sur y $15 \%$ de pendiente. Aparentemente, sin manejo ocupa un suelo de capacidad de uso VI perteneciente a la serie Asociación Pocillas (CIREN 1997). Al momento de extraer la muestra el rodal exhibía una densidad de 692 árboles/ha $(\mathrm{CV}=19,5 \%), 47,9 \mathrm{~m}^{2} / \mathrm{ha}(\mathrm{CV}=24,7 \%)$ de área basal, 25,3 m de altura media de los 100 árboles de mayor DAP $(\mathrm{CV}=5,7 \%)$ y un volumen total de 484 $\mathrm{m}^{3} \mathrm{scc} / \mathrm{ha}(\mathrm{CV}=26,2 \%)$.

La dimensión de los árboles muestra fluctuó entre 25 y $37 \mathrm{~cm}$ de DAP, y 22 y $26 \mathrm{~m}$ de altura total. Los árboles fueron volteados y seccionados en longitudes comerciales de 2,44 m. Las trozas de diámetro mínimo de $20 \mathrm{~cm} \sin$ corteza fueron aserradas y las piezas resultantes encastilladas para su secado al aire. De allí se seleccionó una muestra de 20 piezas de $7 \times 7$ pulgadas $(177,8 \times 177,8 \mathrm{~mm})$ de escuadría y de entre 75 y $90 \mathrm{~cm}$ de largo.

La madera fue seleccionada, predimensionada y acondicionada en cámara de clima a $20 \pm 2^{\circ} \mathrm{C}$ y $65 \pm 5 \%$ de humedad relativa hasta lograr la humedad de equilibrio (INN 1986a). Las probetas definitivas fueron confeccionadas con material de contenido de humedad controlado, permaneciendo en cámara hasta el momento de ser ensayadas. Las determinaciones ${ }^{1}$ se realizaron con un $12 \%$ de contenido de humedad, con apego a las normas chilenas pertinentes a cada tipo de esfuerzo (INN 1986bcdefghi). Los ensayos contemplaron 10 repeticiones en el caso de las propiedades mecánicas: flexión estática, compresión paralela y normal a las fibras, tracción perpendicular, cizalle, clivaje, dureza y extracción de clavos; y 20 para la densidad básica (INN 1988).

Los valores de resistencia de cada esfuerzo fueron correlacionados con la densidad al $12 \%$ de contenido de humedad y se clasificó la madera de acuerdo con los parámetros propuestos por Sallenave (Pérez 1983).

\section{RESULTADOS}

La densidad de la madera de P. canariensis al $12 \%$ de contenido de humedad resultó inferior al rango registrado en España por Peraza y López (1967) para las maderas denominadas pino blanco, riga y tea, en tanto que las determinaciones de Raymond et al. (2004) en Australia superan sólo en 9,3\% a las aquí informadas. Sin embargo, la densidad básica superó levemente el valor promedio máximo informado por Pérez (1983) para $P$. radiata crecido en Chile (cuadro 1).

Análogamente, las cifras de resistencia a la flexión, compresión, tracción perpendicular y dureza de la madera resultaron menores a las proporcionadas por Peraza y López (1967) y Raymond et al. (2004). Con excepción de la resistencia a la tracción perpendicular y el clivaje, la madera de $P$. canariensis es igual o superior a la de $P$. radiata crecida en Chile (Pérez 1983). Como era de esperar (Koch 1972, Kollmann y Coté 1984), las propiedades ensayadas tuvieron una correlación positiva significativa con la densidad de la madera $(P<0,01)$, a excepción de la extracción de clavos (cuadro 2).

Sometidas las variables pertinentes a la metodología de clasificación propuesta por Sallenave, descrita en detalle en Pérez (1983), la madera de pino canario se clasifica según la propiedad como indica el cuadro 3.

\footnotetext{
El análisis se realizó en el Instituto de Tecnología de Productos Forestales de la Facultad de Ciencias Forestales de la Universidad Austral de Chile.
} 
Cuadro 1. Densidad y propiedades mecánicas de la madera de Pinus canariensis.

Pinus canariensis wood density and mechanical properties.

\begin{tabular}{|c|c|c|c|c|c|c|}
\hline \multirow[t]{2}{*}{ Propiedad } & \multirow[t]{2}{*}{ Unidad } & \multirow{2}{*}{$\begin{array}{l}\text { Este estudio } \\
\text { Media } \pm \mathrm{S} \\
\end{array}$} & \multirow{2}{*}{$\begin{array}{c}\text { Peraza y López } \\
\text { (1967) }\end{array}$} & \multirow{2}{*}{$\begin{array}{c}\begin{array}{c}\text { Raymond } e t ~ a l . \\
(2004)\end{array} \\
\text { Media } \\
\end{array}$} & \multicolumn{2}{|c|}{$\begin{array}{c}\text { Pinus radiata } \\
\text { (Pérez 1983) }\end{array}$} \\
\hline & & & & & Mínimo & Máximc \\
\hline Densidad básica & $\mathrm{g} / \mathrm{cm}^{3}$ & $0,503 \pm 0,03$ & - & 0,550 & 0,440 & 0,490 \\
\hline Densidad $12 \%$ & $\mathrm{~g} / \mathrm{cm}^{3}$ & $0,580 \pm 0,04$ & $0,753-1,141$ & - & - & - \\
\hline \multicolumn{7}{|l|}{ Flexión } \\
\hline Módulo de ruptura & MPa & $93,6 \pm 11,9$ & $114,1-147,7$ & 105,9 & 41,2 & 95,9 \\
\hline Módulo de elasticidad & $\mathrm{MPa}$ & $8.336 \pm 733$ & - & 11.970 & 6.300 & 12.600 \\
\hline \multicolumn{7}{|l|}{ Compresión } \\
\hline Paralela & $\mathrm{MPa}$ & $51,1 \pm 5,7$ & $45,9-65,4$ & 55,0 & 30,2 & 46,3 \\
\hline Normal & MPa & $14,8 \pm 1,3$ & - & - & 5,9 & 14,4 \\
\hline \multicolumn{7}{|l|}{ Tracción perpendicular } \\
\hline Tangencial & Mpa & $2,5 \pm 0,2$ & $2,8-3,1$ & - & 4,0 & 4,1 \\
\hline Radial & Mpa & $1,6 \pm 0,1$ & - & - & 2,4 & 2,9 \\
\hline \multicolumn{7}{|l|}{ Cizalle } \\
\hline Tangencial & $\mathrm{MPa}$ & $10,2 \pm 0,6$ & - & - & 7,1 & 12,0 \\
\hline Radial & $\mathrm{MPa}$ & $8,7 \pm 0,4$ & - & - & 5,8 & 10,2 \\
\hline \multicolumn{7}{|l|}{ Clivaje } \\
\hline Tangencial & $\mathrm{N} / \mathrm{mm}$ & $37,7 \pm 2,5$ & - & - & 38 & 76 \\
\hline Radial & $\mathrm{N} / \mathrm{mm}$ & $31,4 \pm 1,7$ & - & - & 28 & 51 \\
\hline \multicolumn{7}{|l|}{ Dureza Janka } \\
\hline Paralela & $\mathrm{N}$ & $4.752 \pm 592$ & - & - & 2.461 & 4.982 \\
\hline Normal & $\mathrm{N}$ & $4.083 \pm 434$ & - & 5.200 & 1.608 & 4.325 \\
\hline \multicolumn{7}{|l|}{ Extracción de clavo } \\
\hline Paralela & $\mathrm{N}$ & $286 \pm 83$ & - & - & 206 & 412 \\
\hline Normal & $\mathrm{N}$ & $309 \pm 29$ & - & - & 275 & 549 \\
\hline
\end{tabular}

Cuadro 2. Relaciones entre propiedades mecánicas y densidad de la madera $\left(\mathrm{D}, \mathrm{g} / \mathrm{cm}^{3}\right)$ al $12 \%$ de humedad. Mechanical properties and wood density at $12 \%\left(\mathrm{D}, \mathrm{g} / \mathrm{cm}^{3}\right)$ moisture relationships.

\begin{tabular}{|c|c|c|}
\hline Propiedad & Ecuación & $\mathrm{R}^{2}$ \\
\hline \multicolumn{3}{|l|}{ Flexión estática (Mpa) } \\
\hline Módulo de ruptura (MOR) & $\mathrm{Rf}=278,25 \mathrm{D}-72,79$ & $0,63 * *$ \\
\hline Módulo de elasticidad (MOE) & $E f=17.271 \mathrm{D}-1.995$ & $0,64 * *$ \\
\hline \multicolumn{3}{|l|}{ Compresión (Mpa) } \\
\hline Paralela & $\mathrm{Rcp}=105,67 \mathrm{D}-9,96$ & $0,80 * *$ \\
\hline Normal tangencial & Rent $=25,08 \mathrm{D}+0,99$ & $0,86 * *$ \\
\hline Normal radial & $\mathrm{Rcnr}=45,98 \mathrm{D}-10,62$ & $0,84 * *$ \\
\hline \multicolumn{3}{|l|}{ Tracción perpendicular (Mpa) } \\
\hline Tangencial & Rtnt $=4,48 \mathrm{D}-0,13$ & $0,77 * *$ \\
\hline Radial & $\mathrm{Rtnr}=2,83 \mathrm{D}-0,02$ & $0,81 * *$ \\
\hline \multicolumn{3}{|l|}{ Cizalle (Mpa) } \\
\hline Tangencial & $R c z t=11,32 D+3,53$ & $0,74 * *$ \\
\hline Radial & Rczr $=8,92 \mathrm{D}+3,52$ & $0,76 * *$ \\
\hline \multicolumn{3}{|l|}{ Clivaje (N/mm) } \\
\hline Tangencial & Rclt $=80,71 \mathrm{D}-9,033$ & $0,65 * *$ \\
\hline Radial & Rclr $=50,49 \mathrm{D}+2,563$ & $0,84 * *$ \\
\hline \multicolumn{3}{|l|}{ Dureza $(\mathrm{N})$} \\
\hline Paralela & $\mathrm{Dp}=17.374 \mathrm{D}-5.326$ & $0,67 * *$ \\
\hline Normal & $\mathrm{Dn}=13.695 \mathrm{D}-3.862$ & $0,73 * *$ \\
\hline \multicolumn{3}{|l|}{ Extracción de clavos $(\mathrm{N})$} \\
\hline Paralela & Recp $=773,42 \mathrm{D}-167,20$ & $0,18 \mathrm{~ns}$ \\
\hline Normal & Recn $=231,57 \mathrm{D}+173,18$ & $0,13 \mathrm{~ns}$ \\
\hline
\end{tabular}

** $P<0,01$; ns: no significativo. 
Cuadro 3. Clasificación de la madera de Pinus canariensis según Sallenave (Pérez 1983).

Pinus canariensis wood classification according Sallenave method (Pérez 1983).

\begin{tabular}{ll}
\hline Propiedad & Clase \\
\hline $\begin{array}{l}\text { Densidad } 12 \% \\
\text { Flexión }\end{array}$ & Semipesada \\
$\quad$ Módulo de ruptura & Mediana \\
Cota de flexión & Grande \\
Compresión paralela & \\
$\quad$ Resistencia a la compresión & Superior \\
Cota de calidad estática & Semipesada \\
Clivaje & \\
Resistencia al clivaje & Pequeña \\
Cota de clivaje & Muy laminable \\
Dureza Janka & \\
Normal a la fibra & Semidura \\
Cota de dureza & Fuerte \\
\hline
\end{tabular}

\section{DISCUSIÓN}

Los menores valores de densidad obtenidos en este estudio respecto de aquellos informados por Peraza y López (1967) pueden ser explicados por la mayor edad de los árboles y diferencias en las condiciones de sitio. La madera analizada por estos autores proviene de árboles maduros de Islas Canarias donde la especie crece en suelos volcánicos profundos, bien drenados, o en suelos de textura franca arcillosa desarrollados sobre arena o granito (Critchfield y Little 1966, Goor y Barney 1976), con precipitaciones en el rango 400-650 mm y 7-8 meses secos por año (Mirov 1967). En tanto que las menores diferencias observadas con los datos de Raymond et al. (2004) responderían a árboles de edad y condiciones de sitio semejantes.

Atendiendo a la estrecha relación existente entre propiedades mecánicas y la densidad de la madera (Koch 1972, Kollmann y Coté 1984), los menores niveles de las propiedades mecánicas determinadas por este estudio, respecto a la información de España y Australia (Peraza y López 1967, Raymond et al. 2004), obedecen precisamente a la menor densidad de la madera aquí informada.

La densidad de la madera de $P$. canariensis determinada en este estudio se ubica en el extremo superior del rango de referencia informado para $P$. radiata crecido en Chile (Pérez 1983). Esta situación permitiría predecir comportamientos semejantes de la madera, de este nivel de densidad, en propiedades de flexión, compresión, cizalle y dureza. Para el caso de la tracción perpendicular, clivaje y extracción de clavo, esta apreciación no se cumple, probablemente a consecuencia del abundante tejido parenquimático de $P$. canariensis en comparación con varias especies del género Pinus, incluido $P$. radiata, que afectaría negativamente a estas propiedades. Lo anterior se explicaría por la mayor presencia de rayos medulares en esta madera, que consti- tuyen planos de debilidad estructural, particularmente en el plano radial (García et al. 2005).

La aplicación de la norma chilena NCh1989, que agrupa a las especies madereras según su resistencia (INN 1989) y que considera como parámetros a los módulos de ruptura y elasticidad, y la resistencia a la compresión paralela (cuadro 1), permite deducir que la madera ensayada corresponde al grupo ES4, superando a $P$. radiata y pino oregón (Pseudotsuga menziesii (Mirb.) Franco), crecidos en Chile que clasifican en ES5. Lo anterior se explicaría por la mayor densidad de $P$. canariensis respecto a las otras coníferas señaladas. Este resultado sitúa a la madera de esta especie al mismo nivel de algunas maderas nativas chilenas como: coigüe (Nothofagus dombeyi (Mirb.) Oerst.), laurel (Laurelia sempervirens (Ruiz et Pav.) Tul.), lenga (Nothofagus pumilio (Poepp. et Endl.) Krasser) y roble (Nothofagus obliqua (Mirb.) Oerst.).

Por otra parte, la aplicación de la clasificación de Sallenave (Pérez 1983) señala que la madera de $P$. canariensis crecida en el secano interior de la Región del Maule en Chile Central es semipesada, apropiada para carpintería, muy laminable y adecuada para usos especiales. Tales indicadores la hacen potencialmente apta para ser empleada en edificación, particularmente en elementos estructurales verticales y horizontales, y para pisos.

\section{CONCLUSIONES}

Los valores de las propiedades físicas y mecánicas determinadas confirman la posibilidad de usar la madera de $P$. canariensis en aplicaciones semejantes a las que ha sido destinada en su lugar de origen, especialmente en la elaboración de elementos estructurales como cerchas, vigas, embalajes y muebles.

Aunque el tamaño de la muestra es limitado, estos resultados estimulan estudios complementarios que consideren madera crecida en otros sitios del país y que amplíen la cantidad de variables analizadas.

\section{REFERENCIAS}

Albert F. 1908. El Pino Tea de las Canarias o Pinus canariensis, Chr. Smith. Anales Agronómicos 3(2 y 3): 333-366.

Barros S. 1997. Ensayos de introducción de especies en la Región Metropolitana. Santiago, Chile. Corporación Nacional Forestal. $61 \mathrm{p}$.

CIREN (Centro de Información de Recursos Naturales, CL). 1997. Estudio agrológico VII Región. Descripciones de Suelos. Materiales y Símbolos. Santiago, Chile. Publicación CIREN No 117. 624 p.

CONAF (Corporación Nacional Forestal, CL). 1990. Centro experimental forestal Tanumé en el desarrollo de Chile Central. Rancagua, Chile. 32 p.

García L, P Gasson, J Climent, P De Palacios, A Guindeo. 2005. The Wood of Pinus canariensis and its resinous heartwood. IAWA Journal 26(1): 69-77. 
Goor AY, CW Barney. 1976. Forest tree planting in arid zones. $2^{\text {nd }}$ ed. New York, USA. The Ronald Press. 504 p.

Critchfield W, E Little. 1966. Geographic distribution of de pines of the world. Washington, USA. U.S. Department of Agriculture. $97 \mathrm{p}$.

Guindeo A, L García. 1988. Anatomía e identificación de las maderas de coníferas españolas. Madrid, España. AITIM. $151 \mathrm{p}$.

INFOR (Instituto Forestal, CL). 1986. Especies forestales exóticas de interés económico para Chile. Santiago, Chile. Corporación de Fomento de la Producción. 168 p.

INN (Instituto Nacional de Normalización, CL). 1986a. Madera - Selección, obtención y acondicionamiento de muestras y probetas para la determinación de propiedades físicas y mecánicas. NCh 968. Of. 1986. 16 p.

INN (Instituto Nacional de Normalización, CL). 1986b. Madera. Determinación de las propiedades mecánicas. Ensayo de flexión estática. NCh 987. Of. 1986. 9 p.

INN (Instituto Nacional de Normalización, CL). 1986c. Madera. Determinación de las propiedades mecánicas. Ensayo de compresión paralela. NCh 973. Of. 1986. 9 p.

INN (Instituto Nacional de Normalización, CL). 1986d. Madera. Determinación de las propiedades mecánicas. Ensayo de compresión perpendicular a las fibras. NCh 974. Of. 1986. 7 p.

INN (Instituto Nacional de Normalización, CL). 1986e. Madera. Determinación de las propiedades mecánicas. Ensayo de tracción perpendicular a las fibras. NCh 975. Of. 1986. $7 \mathrm{p}$.

INN (Instituto Nacional de Normalización, CL). 1986f. Madera. Determinación de las propiedades mecánicas - Ensayo de cizalle paralelo a las fibras. NCh 976.Of. 1986. 8 p.

INN (Instituto Nacional de Normalización, CL). 1986g. Madera. Determinación de las propiedades mecánicas. Ensayo de clivaje. NCh 977. Of. 1986. 7 p.

INN (Instituto Nacional de Normalización, CL). 1986h. Madera. Determinación de las propiedades mecánicas. Ensayo de dureza. NCh 978. Of. 1986. 5 p.

INN (Instituto Nacional de Normalización, CL). 1986i. Madera. Determinación de las propiedades mecánicas. Ensayo de extracción de clavos. NCh 979. Of. 1986. 7 p.
INN (Instituto Nacional de Normalización, CL). 1988. Madera. Determinación de la densidad. NCh 176/2. Of. 1986 MOD.11 p.

INN (Instituto Nacional de Normalización, CL). 1989. Madera. Agrupamiento de especies madereras según su resistencia. Procedimiento. NCh 1989.Of. 1986. Mod 1988. 12 p.

Koch P. 1972. Utilization of southern pines. II. Processing. Washington. U.S. Department of Agriculture Forest Service. Agriculture Handbook No 420. 929 p.

Kollmann F, W Coté. 1984. Principles of wood science and technology. Volume I: Solid Wood. New York, USA. Springer - Verlag. 592 p.

Mirov NT. 1967. The genus pinus. Berkeley, USA. University of California. $602 \mathrm{p}$.

Peraza C, A López.1967. Estudio de las principales maderas de Canarias. Madrid, España. Ministerio de Agricultura. Dirección general de montes, caza y pesca fluvial. Instituto Forestal de Investigaciones y Experiencias, Ministerio de Agricultura. 220 p.

Pérez V. 1983. Manual de propiedades físicas y mecánicas de maderas chilenas. Santiago, Chile. Proyecto CONAF/PNUD/ FAO-CHI 76/003. Documento de Trabajo No 47. 451 p.

Quiroz I, Y Rojas. 2003. Pino Ponderosa \& Pino Oregón. Coníferas para el Sur de Chile. Valdivia, Chile. Instituto Forestal Sede Los Lagos. 302 p.

Raymond C, R Dickson, D Rowell, P Blakemore, N Clark, M Williams, G Freischmidt, B Joe. 2004. Wood and fibre properties of dryland conifers. A report for the RIRDC/L\&W Australia/FWPRDC/MDBC Joint Venture Agroforestry Program. RIRDC Publication $\mathrm{N}^{\circ}$ 04/099. 69 p.

Rodríguez C, J Bobadilla. 2006. Crecimiento inicial de Pinus canariensis en el secano de la VII Región del Maule, Chile. Bosque 27(3): 258-262.

Schlatter J, V Gerding, J Adriazola. 2001. Sistema de ordenamiento de la tierra. Herramienta para la planificación forestal aplicada a las Regiones VII, VIII y IX. Serie Técnica, $3^{\text {a }}$ ed. Valdivia, Chile. Facultad de Ciencias Forestales, Universidad Austral de Chile. 79 p.

Webb D, P Wood, J Smith, G Henman. 1984. A guide to species selection for tropical and sub-tropical plantations. Tropical Forestry Papers $N^{\circ} 15$. $2^{\text {nd }}$ ed. Oxford, Great Britain. Commonwealth Forestry Institute. University of Oxford. 255 p. 